\title{
Cavities of weak magnetic field strength in the wake of FTEs: Results from global magnetospheric MHD simulations
}

\author{
M. M. Kuznetsova, ${ }^{1}$ D. G. Sibeck, ${ }^{1}$ M. Hesse, ${ }^{1}$ Y. Wang, ${ }^{1}$ L. Rastaetter, ${ }^{1}$ G. Toth, ${ }^{2}$ \\ and A. Ridley ${ }^{2}$ \\ Received 26 January 2009; revised 9 April 2009; accepted 20 April 2009; published 22 May 2009.
}

[1] We use the global magnetohydrodynamic (MHD) code BATS-R-US to model multipoint observations of Flux Transfer Event (FTE) signatures. Simulations with high spatial and temporal resolution predict that cavities of weak magnetic field strength protruding into the magnetosphere trail FTEs. These predictions are consistent with recently reported multi-point Cluster observations of traveling magnetopause erosion regions (TMERs). Citation: Kuznetsova, M. M., D. G. Sibeck, M. Hesse, Y. Wang, L. Rastaetter, G. Toth, and A. Ridley (2009), Cavities of weak magnetic field strength in the wake of FTEs: Results from global magnetospheric MHD simulations, Geophys. Res. Lett., 36, L10104, doi:10.1029/2009GL037489.

\section{Introduction}

[2] Russell and Elphic [1978] identified transient bipolar variations of the magnetic field component normal to the nominal magnetopause centered on enhancements in the total magnetic field strength and termed them flux transfer events, or FTEs. The events were originally interpreted as evidence for the passage of elbow-shaped flux tubes that originate via patchy, transient reconnection on the magnetopause. They have also been interpreted in terms of bubbles [Southwood et al., 1988] or flux ropes [Lee and $\mathrm{Fu}, 1985$; Hesse et al., 1990] that form via the reconnection of magnetosheath and magnetospheric magnetic field lines along extended subsolar lines. The strong core magnetic fields have been interpreted in terms of unreconnected magnetosheath magnetic field lines swept up by reconnected magnetosheath-magnetospheric magnetic field lines [Scholer, 1988]. Many FTE events exhibit a layered, or crater-like magnetic field magnitude signature, characterized by an enhancement with a central depression [e.g., Farrugia et al., 1988]. Some crater FTEs exhibit more complicated signatures in which magnetic field strength enhancements bound trenches which in turn bound increases in the field at the center [Sibeck et al., 2008].

[3] Recent Cluster and THEMIS multipoint observations provide new opportunities to study FTE structures. Owen et al. [2001] used Cluster observations to demonstrate the existence of magnetic field compressions ahead of and flow circulation within FTEs. Sonnerup et al. [2004] and Hasegawa et al. [2006] developed a Grad-Shafranov technique based on multipoint observations to reconstruct the cross-sections of FTEs passing over the Cluster spacecraft. They showed that the Cluster event from 0700 to 0715 UT

\footnotetext{
${ }^{1}$ NASA Goddard Space Flight Center, Greenbelt, Maryland, USA.

${ }^{2}$ CSEM, University of Michigan, Ann Arbor, Michigan, USA.
}

Copyright 2009 by the American Geophysical Union. 0094-8276/09/2009GL037489 on March 8, 2003 exhibited a nearly circular cross section with a strong core magnetic field. Lui et al. [2008] used the same technique to derive a circular cross-section for a THEMIS event reported by Sibeck et al. [2008]. The region of enhanced magnetic field strengths within the core region of this crater FTE lies within a broadened magnetopause current layer with weak magnetic field strengths.

[4] Owen et al. [2008] recently interpreted multipoint Cluster observations from 06:40 to 07:10 UT on January 11, 2002 as evidence for the passage of a region of eroded magnetic flux trailing FTEs. Cluster-1 was one of three spacecraft that detected bipolar Bn signatures normal to the nominal magnetopause and crater-like variations in the total magnetic field strength at 06:50 and 0658 UT: the signatures that typify crater FTEs. Cluster-1 remained in the magnetosphere before, during, and after these events until 07:02 UT, when it encountered the first of several magnetopause crossings. Cluster-3 began the interval in the magnetosphere, observed an enhanced magnetospheric magnetic field strength at 06:50 UT, a bipolar signature normal to the nominal magnetopause centered on a magnetopause current layer at 06:50:30 UT, and then briefly exited from the magnetosphere into the magnetosheath to observe weaker and more variable magnetic field strengths and greatly enhanced densities. Owen et al. termed this wake region of weak magnetosheath magnetic field strengths a 'traveling magnetopause erosion region' or TMER. Cluster3 observed a similar, but far less-pronounced, sequence of events at 0658 UT, the time of the second FTE seen by Cluster-1. To interpret the observations Owen et al. [2008] suggested that FTE is moving duskward and poleward in the direction of the DeHoffman-Teller vector, while the erosion region (TMER) is driven in the direction of the magnetosheath flow. Therefore the TMER is being convected duskward like the FTE, but moving poleward less rapidly.

[5] In this paper, we examine the output of a global magnetohydrodynamic (MHD) simulation for evidence of TMERs. Several studies have already demonstrated that transients with the signatures of FTEs are common in the output from high time and spatial resolution global MHD models [e.g., Fedder et al., 2002; Kuznetsova et al., 2004; Raeder, 2006]. Most recently, Sibeck et al. [2008] employed the BATS-R-US code [Powell et al., 1999] to simulate a single crater FTE observed by the THEMIS spacecraft. There was no TMER associated with this event in either the observations or the simulation output. However, the MHD run in which this event was embedded covered a full three hours of relatively steady duskward and southward IMF orientations. The simulation predicted numerous FTEs exhibiting a wide range of signatures and dimensions. This 

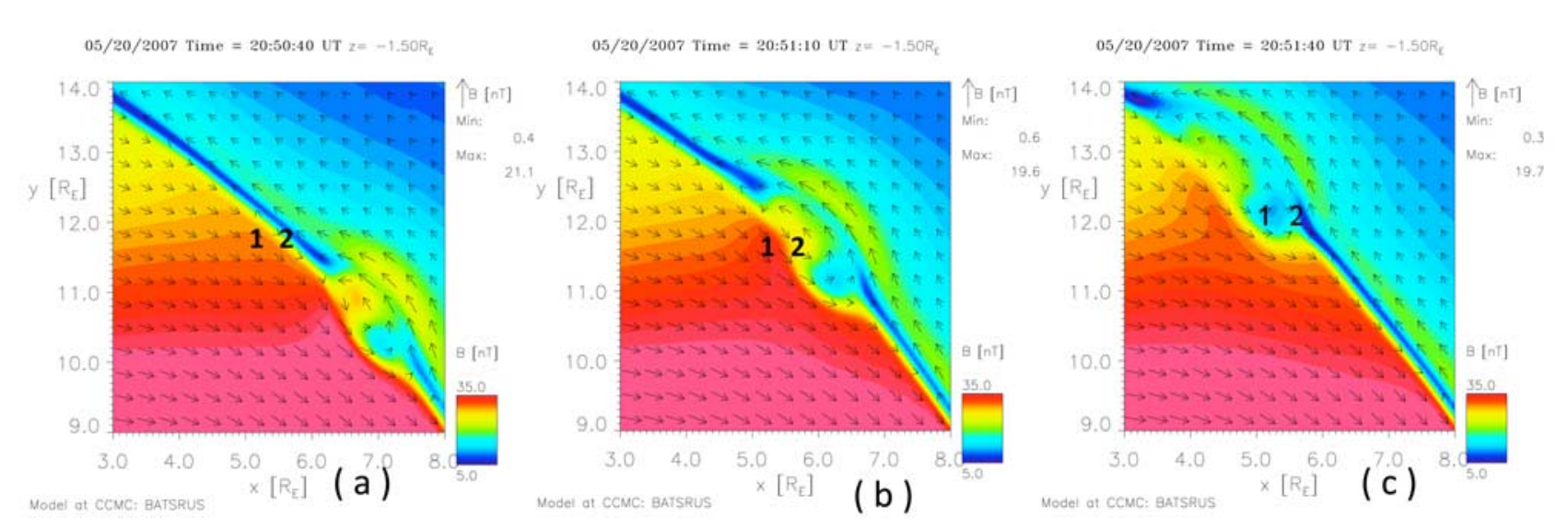

Figure 1. Magnetic field strengths and vectors in post-noon cuts through the GSM $Z=-1.5 R_{E}$ plane at three time steps separated by 30 seconds, (a) 20:50:40, (b) 20:51:10, and (c) 20:51:40.

study focuses on just one of the FTEs predicted by the model, an event in which the FTE was followed by a cavity of weak magnetic field strengths. We demonstrate that the simulation results for this event are qualitatively consistent with the scenario recently reported by Owen et al. [2008].

\section{Simulation Setup}

[6] We employ the global MHD code BATS-R-US developed by the Center for Space Environment Modeling at the University of Michigan [Powell et al., 1999; Ridley et al., 2004; Toth et al., 2005]. The relatively steady solar wind magnetic field and plasma parameters observed by ACE on May 20, 2007, from 20:00 to 23:00 UT are propagated with the average solar wind speed to the inflow boundary of the GM domain at $X=33 R_{E}$. The simulation box size in GSM coordinates is $-255 \mathrm{R}_{\mathrm{E}}<\mathrm{X}<33 \mathrm{R}_{\mathrm{E}},|\mathrm{Y}|<48 \mathrm{R}_{\mathrm{E}},|\mathrm{Z}|<48 \mathrm{R}_{\mathrm{E}}$.

[7] The block-based adaptive domain-decomposition technique implemented in BATS-R-US permits construction of a simulation grid with about $20,000,000$ cells, including a highly resolved region $\left(\Delta \mathrm{X}=\Delta \mathrm{Y}=\Delta \mathrm{Z}=1 / 16 \mathrm{R}_{\mathrm{E}}\right)$ wrapped around the entire magnetopause boundary layer up to the terminator. The uniformly resolved grid in the vicinity of the magnetopause permits us to track FTEs from formation in the subsolar region to the terminator without any significant diffusive smearing of their internal structure. The run is performed with the low-diffusivity Sokolov numerical scheme [Sokolov et al., 2002]. During the time interval of interest in this study the model output is saved every 10 seconds.

\section{Simulation Results}

[8] Figures $1 \mathrm{a}, 1 \mathrm{~b}$, and $1 \mathrm{c}$ show $\mathrm{Z}=-1.5 \mathrm{R}_{\mathrm{E}}$ cuts through the simulation domain "zoomed-in" to the region at the dusk flank of the magnetopause at three times (20:50:40, 20:51:10, and 20:51:40 UT). The X-Y dimensions of the zoomed-in slice centered around $X=5.5 R_{E}$, $\mathrm{Y}=11.5 \mathrm{R}_{\mathrm{E}}$ are $5 \mathrm{R}_{\mathrm{E}}$, much less than the size of the global simulation domain. Colors in Figure 1 display the total magnetic field strength, while arrows depict the projection of the magnetic field vectors onto the cut plane.

[9] The numbers " 1 " and " 2 " in Figure 1 indicate the locations of two virtual probes near the magnetopause at
$\left(\mathrm{X}=5.25 \mathrm{R}_{\mathrm{E}}\right.$ and $\left.5.75 \mathrm{R}_{\mathrm{E}}, \mathrm{Y}=11.8 \mathrm{R}_{\mathrm{E}}, \mathrm{Z}=-1.5 \mathrm{R}_{\mathrm{E}}\right)$ respectively. At 20:50:40 UT, the two virtual spacecraft bound the undisturbed magnetopause. Probe 2 lies in the magnetopause layer of depressed $(10-15 \mathrm{nT})$ magnetic field strengths that separates northward, sunward, and dawnward magnetic fields in the magnetosphere on the lower left from southward, antisunward, and duskward magnetic fields in the magnetosheath on the upper right. Probe 1 lies just inside the magnetosphere, where it observes strong ( $30 \mathrm{nT}$ ) dawnward and northward magnetic fields. Magnetosheath magnetic field strengths diminish from $\sim 30 \mathrm{nT}$ in a thin 'pile-up' region just outside the magnetopause to $10 \mathrm{nT}$ further away. An FTE with $\sim 25 \mathrm{nT}$ core magnetic field strengths that bulges into the magnetosheath and magnetosphere near $(x, y, z)=(6.8,10.8,-1.5) R_{E}$ can be seen in the right lower corner of Figure 1a.

[10] From 20:51:00 to 20:51:10 UT (Figure 1b), the enhanced magnetic field strengths within the core of the duskward-moving FTE pass the virtual spacecraft. Magnetic field lines within this FTE spiral about an axis that locally runs from southern dawn to northern dusk, consistent with the expected orientation of a component reconnection line during this interval of southward and duskward magnetosheath magnetic fields. Magnetosheath magnetic field strengths are also greatly enhanced within the region of magnetosheath magnetic field lines that drape over the FTE. Virtual spacecraft 1 remains just inside the magnetosphere while the core of the FTE passes over virtual spacecraft 2.

[11] By 20:51:40 UT, the enhanced magnetic field strength region within the core of the FTE has passed both spacecraft, leaving them in the broadened current layer of weak magnetic field strengths lying within the wake of the FTE (Figure 1c). Interestingly, virtual spacecraft 1 enters this region without ever encountering the enhanced magnetic field strengths within the core region of the FTE. This happens because the region of weak magnetic field strengths trailing the FTE protrudes into the magnetosphere.

[12] Comparing the FTE core and magnetic cavity center positions on $Z=-1.5 R_{E}$ plane at different times (Figures 1a, $1 \mathrm{~b}$, and 1c) one can estimate an average duskward speed of $200 \mathrm{~km} / \mathrm{s}$. The magnetic cavity follows the FTE core duskward motion in the $\mathrm{X}-\mathrm{Y}$ plane.

[13] Figures 2a, 2b, 2c, and 2d show magnetic field strength, density, pressure and Z-component of the plasma 
05/20/2007 Time $=20: 51: 10$ UT $z=-1.50 R_{E}$

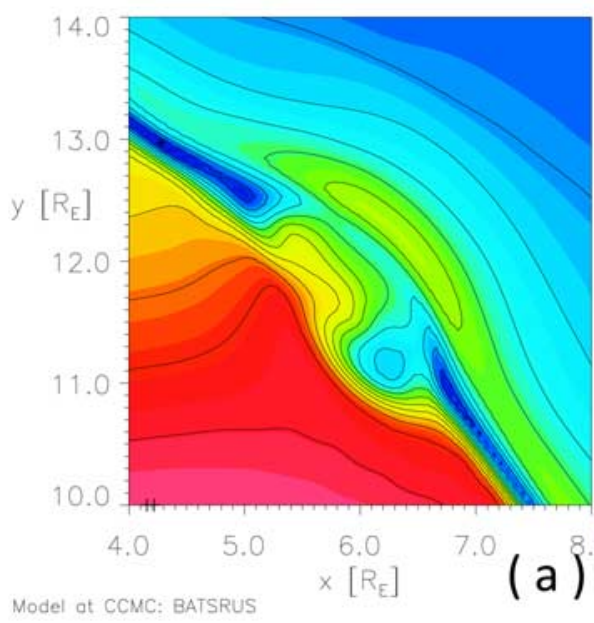

$05 / 20 / 2007$ Time $=20: 51: 10$ UT $z=-1.50 R_{E}$

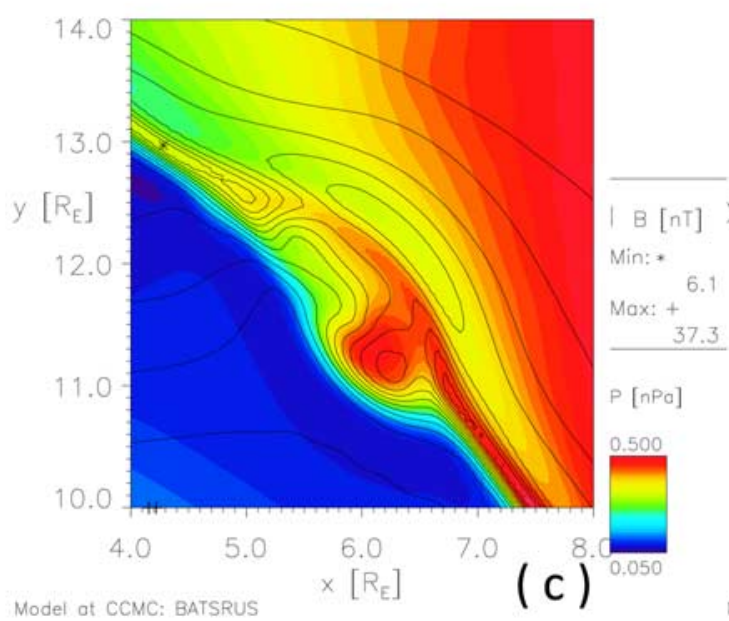

$05 / 20 / 2007$ Time $=20: 51: 10$ UT $z=-1.50 R_{\mathrm{E}}$

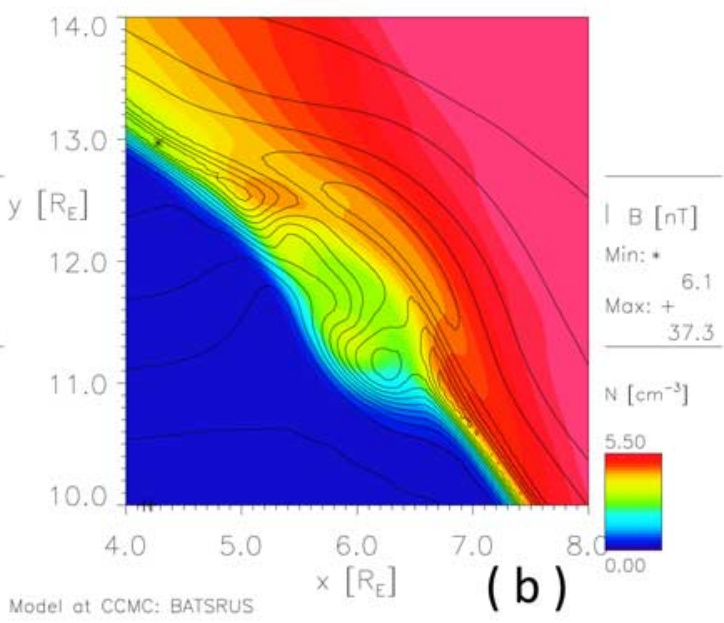

$05 / 20 / 2007$ Time $=20: 51: 10$ UT $z=-1.50 R_{E}$

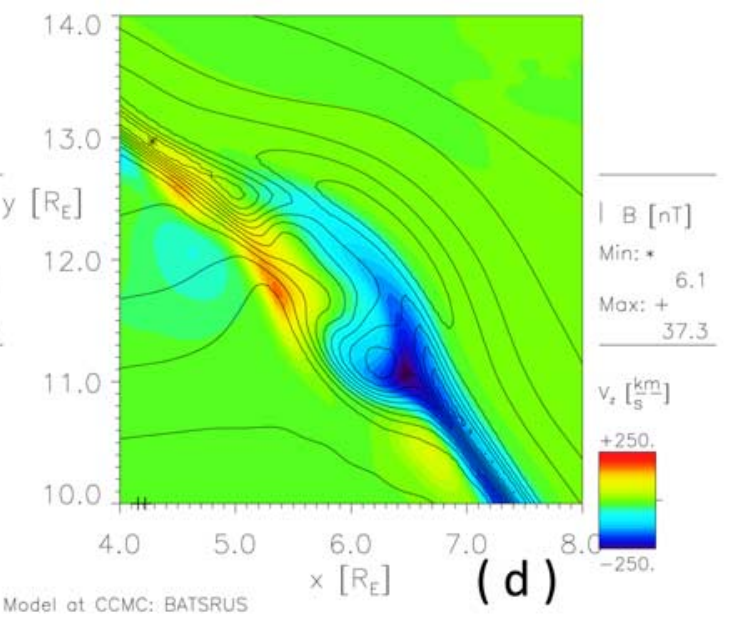

Figure 2. (a) Magnetic field strength, (b) density, (c) pressure, and (d) Z-component of the plasma velocity distributions $\mathrm{VZ}$ in post-noon cuts through the GSM Z $=-1.5 \mathrm{R}_{\mathrm{E}}$ plane for the same time step as Figure $1 \mathrm{~b}(20: 51: 10)$.

velocity distributions in the $\mathrm{Z}=-1.5 \mathrm{R}_{\mathrm{E}}$ slice centered on the FTE at 20:51:10 UT. Contour lines correspond to magnetic field strength. The density in the FTE core as well as within the wake of the FTE is significantly larger than in the magnetosphere, which is consistent with Cluster-3 observations on January 112002 at 06:50:30 UT during its brief excursion from the magnetosphere to the magnetosheath [Owen et al., 2008]. Density values in the simulated FTE core $(x, y, z)=(5.7,11.8,-1.5)$ and in the wake of the FTE $(\mathrm{x}, \mathrm{y}, \mathrm{z})=(6.3,11.2,-1.5)$ are comparable and fall between the density values in the magnetosphere and in the magnetosheath. The plasma pressure in the wake of the FTE is larger than in the FTE core to compensate for the reduced magnetic field strength and pressure. The pressure increase is supported by an increase in the plasma temperature, not of the density. The plasma flow velocity $\mathrm{Vz}$ in the north-south direction (Figure 2d) is significantly different in the region of enhanced core field strength and in the center of the magnetic cavity.

[14] Figure 3 shows a perspective view of magnetic field lines traced from the $Z=-1.5 R_{E}$ slice. Yellow lines that cross the $Z=-1.5 R_{E}$ slice at the center of the enhanced field strength region are magnetic field lines with both open ends connected to the colder plasma in the magnetosheath. The northern ends of red (closed) and grey (semi-open) field lines that pass through the center of the magnetic cavity in the $\mathrm{Z}=-1.5 \mathrm{R}_{\mathrm{E}}$ slice are connected to the hotter magnetosphere plasma and to the northern polar region. The magnetic field lines that pass through the magnetic cavity spiral around the field lines crossing the strong field region. Both types of field lines originated via repeated magnetic reconnection. The field lines crossing the core region remain open for longer periods of time, so they contain more cold magnetosheath plasma. The differing magnetic connections explain the plasma density and pressure distributions shown in Figures $2 \mathrm{~b}$ and $2 \mathrm{c}$. Colder plasma in the strong field region is also consistent with a model of core field generation during plasmoid evolution suggested by Hesse et al. [1996]. There is no evidence that the strong field region is formed by rolling up unreconnected interplanetary magnetic field lines.

[15] Figure 4a presents magnetic field strengths (solid curves) and magnetic field components normal to the nominal magnetopause (dashed curves) at the two virtual 
probes from 20:50 to 20:53 UT. Consider Probe 2 (blue) first. This probe began and ended the interval in the magnetopause current layer just outside the magnetosphere, where it observed weak magnetic field strengths $(8-18 \mathrm{nT})$. The spike in magnetic field strength to $24 \mathrm{nT}$ at 20:51:10 UT corresponded to the encounter with the enhanced magnetic field strengths within the core region of the FTE. This spike was bounded by weak $(\sim 8 \mathrm{nT})$ magnetic field strengths. Probe 2 observed a bipolar $(-,+) B_{n}$ magnetic field signature normal to the nominal magnetopause that lay centered on this magnetic field strength enhancement. We conclude that Probe 2 observed an FTE characterized by bipolar magnetic field lines spiraling around a strong core magnetic field bounded by weaker magnetic fields.

[16] Now consider the signatures observed by Probe 1 (red). This probe observed strong $(\sim 30 \mathrm{nT})$ magnetic field strengths within the magnetosphere at the beginning and end of the interval. It observed a slight increase in the magnetic field strength at 20:51:10 UT, the magnetopause current layer at 20:51:20 UT, and a dramatic weakening in the total magnetic field strength by a factor of 2 to $\sim 15 \mathrm{nT}$ at 20:51:30 UT, before reentering the magnetosphere via the current layer at 20:51:40 UT. Probe 1 observed a bipolar signature in the magnetic field component normal to the nominal magnetopause (red dashed curve) centered on the first crossing of the magnetopause current layer at 20:51:20 UT.

[17] Probe 1's position with respect to the magnetopause in our simulations is similar to Cluster-3's position on January 12,2002 . We equate the signatures observed by Probe 1 to those observed by Cluster-3 [Owen et al., 2008]. Both spacecraft were initially located just inside the magnetopause, where they observed transient compressions, and then briefly exited the magnetosphere to observe weak magnetic field strengths. The results shown in Figure 4a

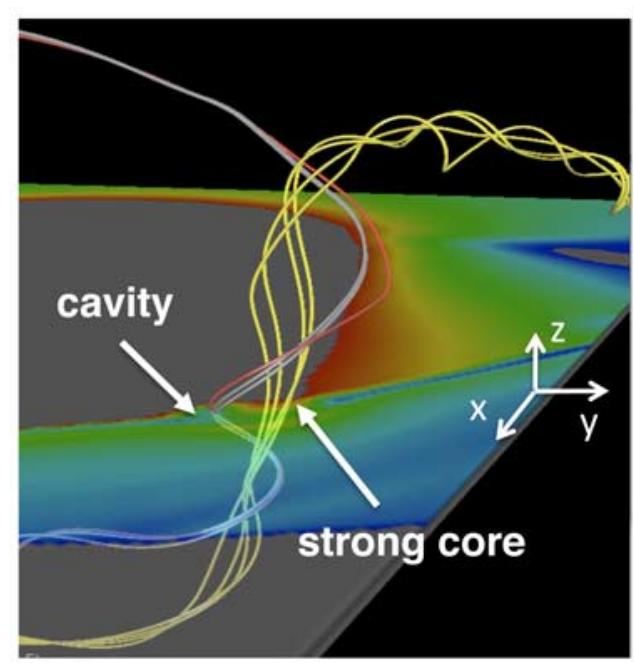

Figure 3. Perspective view of magnetic field lines traced from the center of the strong core region and from the center of the magnetic cavity in the $Z=-1.5 \mathrm{R}_{\mathrm{E}}$ plane. Yellow lines are open field lines with both ends connected to interplanetary space, red lines are closed field lines connecting northern and southern polar regions, and grey lines are semi-open fieled lines connections polar regions to interplanetary space.
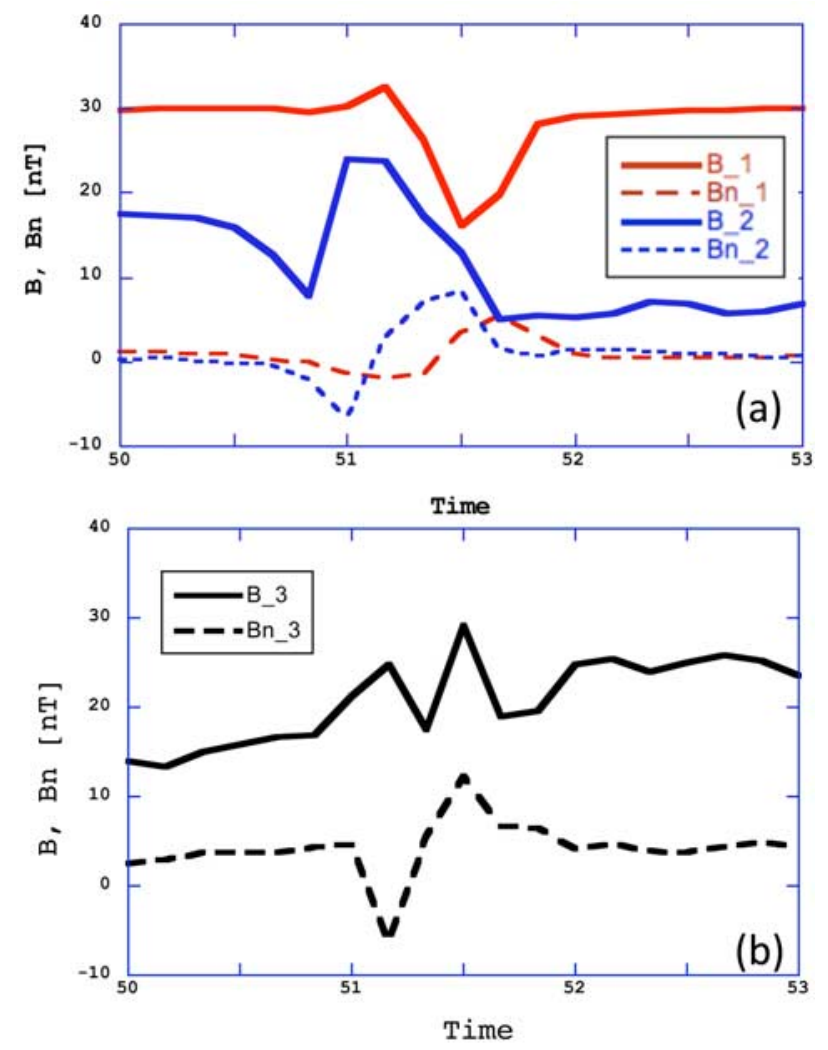

Figure 4. Variations in the magnetic field strength (solid curves) and magnetic field component normal to the nominal magnetopause (dashed curves): (a) at probes 1 and 2 positioned at $\mathrm{z}=-1.5 \mathrm{R}_{\mathrm{E}}$ plane shown in Figure 1; (b) at probe 3 located at $(\mathrm{x}, \mathrm{y}, \mathrm{z})=(4.5,12.3,-3) \mathrm{R}_{\mathrm{E}}$ Time is in minutes after 20:00:00 UT, May 20, 2007.

indicate that this excursion is due to the passage of a region of depressed magnetic field strengths trailing the FTE. This region represents a broadened magnetopause current layer and bulges into the magnetosphere.

[18] Probe 2's position in the current layer was chosen for comparison with THEMIS observations and does not correspond to the position of Cluster-1 deeper in the magnetosphere. Figure $4 \mathrm{~b}$ presents the magnetic field strength and magnetic field component normal to the nominal magnetopause for virtual Probe $3(\mathrm{x}, \mathrm{y}, \mathrm{z})=(4.5,12.3,-3) \mathrm{R}_{\mathrm{E}}$ located about $1 \mathrm{R}_{\mathrm{E}}$ earthward from the magnetopause and about 1.5 $\mathrm{R}_{\mathrm{E}}$ poleward from Probe 1. The magnetic field profiles observed by Probe 3 are similar to the crater FTE signatures characterized by enhancement with a central depression observed by Cluster-1.

\section{Summary}

[19] This paper was motivated by recently reported observations indicating that spacecraft located just inside the magnetopause may emerge into the magnetosheath to observe weak magnetic field strengths in the wake of FTEs. We have located precisely such signatures in the output from the accurate high-time and -spatial resolution BATS-R-US model. We find that the exit from the magnetosphere results from the passage of an inward bulge in the magnetopause current layer trailing an FTE with a strong core magnetic 
field. The density within the bulge exceeds the density in the magnetosphere and is comparable to the density in the FTE core. Pressure and temperature in the trailing magnetic cavity are larger than in the FTE core. We have checked the hypothesis put forward by Owen et al. [2008] that the trailing region of depressed magnetic field strengths moves in a direction that differs from that of the FTE itself. We found that the magnetic cavity and the strong core FTE move together towards the flanks with an average duskward speed of $200 \mathrm{~km} / \mathrm{s}$. The flow speed in the north-south direction is significantly different in the region of enhanced core field strength and in the center of the magnetic cavity. This flow speed difference can be an indication of reshaping of the flux tube and the redistribution of plasma and magnetic field within it. However there is no direct evidence that the strong core region and magnetic cavity are drifting apart. Virtual Probes in the simulation reported here were set close to THEMIS spacecraft positions near the equatorial plane on May 20, 2007, while Cluster FTE and TMER observations reported by Owen et al. [2008] were at higher latitude. Difference in flow pattern near the equatorial plane and at high latitudes could affect cavity and FTE motion. Further dedicated studies of these phenomena will include simulations of the Cluster January 12, 2002 event.

[20] The events discussed here, and by Sibeck et al. [2008], represent only two of a wide variety of transient events seen within a single run of the numerical simulation for real solar wind parameters. Some events do not exhibit strong core magnetic field strengths, while others do not exhibit the bulging current sheets. The reasons for these differences remain unclear, but may stem from differing magnetic field configurations (e.g., shears or field strengths) at the point of reconnection. Although we have not shown it here, we should also note that the characteristics of individual FTEs differ greatly along their axes. A crater FTE at one location may be connected to an FTE with a weak core magnetic field elsewhere. The three-dimensional structure of FTE flux tubes will be addressed in a forthcoming publication.

[21] Acknowledgments. Calculations were performed at the Community Coordinated Modeling Center (CCMC) as part of a science-based validation activity. Simulation code BATS-R-US was developed by the Center for the Environment Modeling (CSEM) at the University of Michigan. Visualization tools used in this paper were developed by Lutz Rastaetter and David Berrios at CCMC.

\section{References}

Farrugia, C. J., R. C. Elphic, D. J. Southwood, and S. W. H. Cowley (1988), Field and flow perturbations outside the reconnected field line region in flux transfer events: Theory, Planet. Space Sci., 35, 227-240.
Fedder, J. A., S. P. Slinker, J. G. Lyon, and C. T. Russell (2002), Flux transfer events in global numerical simulations of the magnetosphere, J. Geophys. Res., 107(A5), 1048, doi:10.1029/2001JA000025.

Hasegawa, H., B. U. Ö. Sonnerup, C. J. Owen, B. Klecker, G. Paschmann, A. Balogh, and H. Rème (2006), The structure of flux transfer events recovered from Cluster data, Ann. Geophys., 24, 603-618.

Hesse, M., J. Birn, and K. Schindler (1990), On the topology of flux transfer events, J. Geophys. Res., 95, 6549-6560.

Hesse, M., J. Birn, M. M. Kuznetsova, and J. Dreher (1996), A simple model of core field generation during plasmoid evolution, J. Geophys. Res., 101, 10,797-10,804

Kuznetsova, M. M., M. Hesse, L. Rastaetter, and T. Gombosi (2004), Intermittent reconnection, flux ropes and vortices generation at the dayside magnetopause, Eos Trans. AGU, 85(47), Fall Meet. Suppl., Abstract SM51C-0387.

Lee, L.-C., and Z.-F. Fu (1985), A theory of magnetic flux transfer at the Earth's magnetopause, Geophys. Res. Lett., 12, 105-108.

Lui, A. T. Y., D. G. Sibeck, T. Phan, V. Angelopoulos, J. McFadden, C. Carlson, D. Larson, J. Bonnell, K.-H. Glassmeier, and S. Frey (2008), Reconstruction of a magnetic flux rope from THEMIS observations, Geophys. Res. Lett., 35, L17S05, doi:10.1029/2007GL032933.

Owen, C. J., et al. (2001), CLUSTER PEACE observations of electrons during magnetospheric flux transfer events, Ann. Geophys., 19, 1509-1522.

Owen, C. J., A. Marchaudon, M. W. Dunlop, A. N. Fazakerley, J.-M. Bosqued, J. P. Dewhurst, R. C. Fear, S. A. Fuselier, A. Balogh, and H. Rème (2008), Cluster observations of "crater" flux transfer events at the dayside high-latitude magnetopause, J. Geophys. Res., 113, A07S04, doi:10.1029/2007JA012701.

Powell, K. G., P. L. Roe, T. J. Linde, T. I. Gombosi, and D. L. De Zeeuw (1999), A solution adaptive upwind scheme for ideal magnetohydrodynamics, J. Comput. Phys., 154, 284-309.

Raeder, J. (2006), Flux transfer events: 1. Generation mechanism for strong southward IMF, Ann. Geophys., 24, 381-392.

Ridley, A., T. Gombosi, and D. L. De Zeeuw (2004), Ionospheric control of the magnetosphere: Conductance, Ann. Geophys., 22, 567-584.

Russell, C. T., and R. C. Elphic (1978), Initial ISEE magnetometer results: Magnetopause observations, Space Sci. Rev., 22, 681-715.

Scholer, M. (1988), Strong core magnetic fields in magnetopause flux transfer events, Geophys. Res. Lett., 15, 748-751.

Sibeck, D. G., M. Kuznetsova, V. Angelopoulos, K.-H. Glaßmeier, and J. P. McFadden (2008), Crater FTEs: Simulation results and THEMIS observations, Geophys. Res. Lett., 35, L17S06, doi:10.1029/2008GL033568.

Sokolov, I. V., E. V. Timofeev, J. I. Sakai, and K. Takayama (2002), Artificial wind: A new framework to construct simple and efficient shock capturing schemes, J. Comput. Phys., 181, 354-393.

Sonnerup, B. U. Ö., H. Hasegawa, and G. Paschmann (2004), Anatomy of a flux transfer event seen by Cluster, Geophys. Res. Lett., 31, L11803, doi:10.1029/2004GL020134.

Southwood, D. J., C. J. Farrugia, and M. A. Saunders (1988), What are flux transfer events?, Planet. Space Sci., 36, 503-508.

Toth, G., et al. (2005), Space Weather Modeling Framework: A new tool for the space science community, J. Geophys. Res., 110, A12226, doi:10.1029/ 2005JA011126.

M. Hesse, M. M. Kuznetsova, L. Rastaetter, D. G. Sibeck, and Y. Wang, NASA Goddard Space Flight Center, Code 674, Greenbelt, MD 20771, USA. (maria.m.kuznetsova@nasa.gov)

A. Ridley and G. Toth, CSEM, University of Michigan, 2455 Hayward Street, Ann Arbor, MI 48109-2143, USA. 